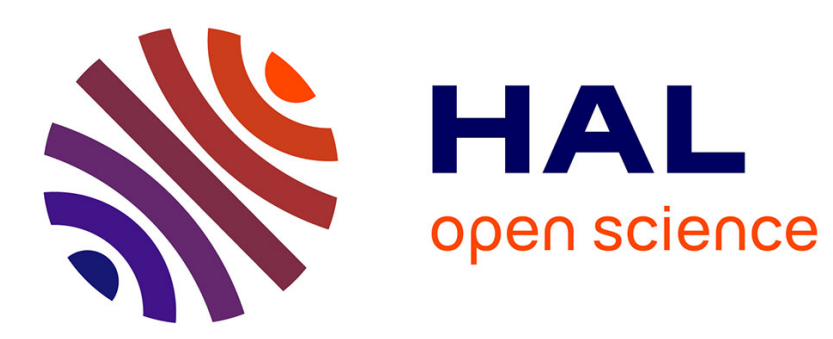

\title{
On Some Special Substructures of Refined Neutrosophic Rings
}

\author{
Mohammad Abobala
}

\section{To cite this version:}

Mohammad Abobala. On Some Special Substructures of Refined Neutrosophic Rings. International Journal of Neutrosophic Science, 2020, 5, pp.59 - 66. 10.5281/zenodo.3789325 . hal-02876518

\section{HAL Id: hal-02876518 https://hal.science/hal-02876518}

Submitted on 10 Aug 2020

HAL is a multi-disciplinary open access archive for the deposit and dissemination of scientific research documents, whether they are published or not. The documents may come from teaching and research institutions in France or abroad, or from public or private research centers.
L'archive ouverte pluridisciplinaire HAL, est destinée au dépôt et à la diffusion de documents scientifiques de niveau recherche, publiés ou non, émanant des établissements d'enseignement et de recherche français ou étrangers, des laboratoires publics ou privés. 


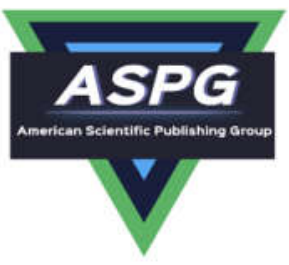

On Some Special Substructures of Refined Neutrosophic Rings

\author{
Mohammad Abobala \\ Tishreen University, Faculty of Science, Lattakia, Syria \\ e-mail: mohammadabobala777@gmail.com
}

\begin{abstract}
The objective of this article is to define and study the concepts of refined neurosophic AH-ideal and AHS-ideal in refined neutrosophic rings. We investigate the elementary properties of these concepts.
\end{abstract}

Keywords: Refined neutrosophic ring, Refined neutrosophic AH-ideal, Refined neutrosophicAHS-Ideal, Refined AHS-homomorphism.

\title{
1.Introduction
}

Neutrosophy as a new branch of philosophy can be applied into the algebraic systems, which leads to a better comprehension and evolution of these systems. The notion of neutrosophic groups and rings was defined by Kandasamy and Smarandache in [10], and studied widely in [4, 5, 7,8]. Studies were carried out on neutrosophic rings and neutrosophic hyperring. See[1, 3, 4,6].

Refined neutrosophic rings were defined and studied carefully in [2, 3], where special substructures such as refined neutrosophic subrings and refined neutrosophic ideals are defined. Many interesting results were proved. In [1] concepts as AH-ideal and AHS-ideal were defined and studied as interesting substructures of neutrosophic ring. Some related concepts such as weak principal, maximal, and prime AH-ideals were introduced. These concepts have many properties which are similar to classical case of rings.In this paper, we try to define concepts such as AH-ideal and AHS-ideal in refined neutrosophic ring with some related concepts such as weak prime, principal and maximal refined neutrosophic AH-ideals. Also, we introduce the notion of refined AHS-homomorphism in similar way to AHS-homomorphism defined in [1].

\section{Motivation}

This paper is the continuation of the work began in the paper entitled "On Some Special Substructures of Neutrosophic Rings and Their properties".

\section{Preliminaries}

\section{Definition 2.1:[5]}

Let $(\mathrm{R},+, \mathrm{X})$ be a ring then $\mathrm{R}(\mathrm{I})=\{\mathrm{a}+\mathrm{bI} ; \mathrm{a}, \mathrm{b} \in R\}$ is called the neutrosophic ring where $\mathrm{I}$ is a neutrosophic element with the condition $I^{2}=I$. 
Remark 2.2: [2]

The element I can be splitinto two indeterminacies $I_{1}, I_{2}$ with conditions:

$$
\mathrm{I}_{1}^{2}=\mathrm{I}_{1}, I_{2}^{2}=I_{2}, I_{1} I_{2}=I_{2} I_{1}=I_{1} .
$$

Definition 2.3: [2]

If $\mathrm{X}$ is a set then $\mathrm{X}\left(I_{1}, I_{2}\right)=\left\{\left(a, b I_{1}, c I_{2}\right) ; a, b, c \in X\right\}$ is called the refined neutrosophic set generated by $\mathrm{X}, I_{1}, I_{2}$.

\section{Definition 2.4: [2]}

Let $(\mathrm{R},+, \times)$ be a ring, $\left(\mathrm{R}\left(I_{1}, I_{2}\right),+, \times\right)$ is called a refined neutrosophic ring generated by $\mathrm{R}, I_{1}, I_{2}$.

\section{Definition 2.5: [2]}

Let $\left(\mathrm{R}\left(I_{1}, I_{2}\right),+, \times\right)$ be a refined neutrosophic ring; it is called commutative if

$$
x \times y=y \times x, \forall x, y \in \mathrm{R}\left(I_{1}, I_{2}\right) .
$$

Theorem 2.6: [2]

Let $\left(\mathrm{R}\left(I_{1}, I_{2}\right),+, \times\right)$ be a refined neutrosophic ring then it is a ring.

\section{Definition 2.7: [3]}

Let $\left(\mathrm{R}\left(I_{1}, I_{2}\right),+, \times\right)$ be a refined neutrosophic ring and $\mathrm{J}$ be a nonempty subset of $\mathrm{R}\left(I_{1}, I_{2}\right)$ then $\mathrm{J}$ is called a neutrosophic refined ideal if:

(a) $\mathrm{J}$ is a refined neutrosophic subring of $\mathrm{R}\left(I_{1}, I_{2}\right)$.

(b) For every $x \in J$ and $r \in \mathrm{R}\left(I_{1}, I_{2}\right)$ then $x \times r \in \mathrm{R}\left(I_{1}, I_{2}\right)$.

\section{Definition 2.8:[1]}

Let $\mathrm{R}(\mathrm{I})$ be a neutrosophic ring and $P=P_{0}+P_{1} I=\left\{a_{0}+a_{1} I ; a_{0} \in P_{0}, a_{1} \in P_{1}\right\}$.

(a)We say that $\mathrm{P}$ is an $\mathrm{AH}$-ideal if $P_{0}, P_{1}$ are ideals in the ring $\mathrm{R}$.

(b)We say that $\mathrm{P}$ is an AHS-ideal if $P_{0}=P_{1}$.

\section{Definition 2.9:[1]}

Let $\mathrm{R}(\mathrm{I})$, $\mathrm{T}(\mathrm{J})$ be two neutrosophic rings and the map $f: R(I) \rightarrow T(J)$; we say that $\mathrm{f}$ is a neutrosophic AHShomomrphism :

Restriction of the map $f$ on $\mathrm{R}$ is a ring homomorphism from $\mathrm{R}$ to $\mathrm{T}$, i.e $f_{R}: R \rightarrow T$ is homomorphism and $f(a+b \mathrm{I})=f_{R}(a)+f_{R}(b) J$

We say that $\mathrm{R}(\mathrm{I}), \mathrm{T}(\mathrm{J})$ are AHS-isomomrphic neutrosophic rings if there is a neutrosophic AHS-homomorphism 
$f: R(I) \rightarrow T(J)$ which is a bijective map; i.e $(\mathrm{R} \cong T)$, we say that $\mathrm{f}$ is a neutrosophic AHS-isomorphism.

\section{Definition 2.10:[1]}

Let $\mathrm{R}(\mathrm{I})$ be a neutrosophic ring and $P=P_{0}+P_{1} I$ be an AH-ideal, we define the AH-factor as:

$R(I) / P=R / P_{0}+R / P_{1} I$.

\section{Theorem 2.11:[1]}

Let $\mathrm{R}(\mathrm{I})$ be a neutrosophic ring and $P=P_{0}+P_{1} I$ be an AH-ideal then $R(I) / P$ is a ring.

Theorem 2.12:[1]

Let $\mathrm{R}(\mathrm{I}), \mathrm{T}(\mathrm{J})$ be two neutrosophic rings and $\mathrm{f}: \mathrm{R}(\mathrm{I}) \rightarrow \mathrm{T}(\mathrm{J})$ is a neutrosophic ring AHS-homomorphism, let $P=P_{0}+$ $P_{1} I$ be an AH-ideal of $\mathrm{R}(\mathrm{I})$ and $Q=Q_{0}+Q_{1} J$ be an AH-ideal of $\mathrm{T}(\mathrm{J})$, we have:

(a) $f(P)$ is an AH-ideal of $f(R(I))$.

(b) $f^{-1}(Q)$ is an AH-ideal of $\mathrm{R}(\mathrm{I})$.

(c) If $\mathrm{P}$ ia an AHS-ideal of $\mathrm{R}(\mathrm{I}), \mathrm{f}(\mathrm{P})$ is an AHS-ideal of $\mathrm{f}(\mathrm{R}(\mathrm{I}))$.

(d) $A H-\operatorname{ker} f=\operatorname{ker} f_{R}+\operatorname{ker} f_{R} I$ is an AHS-ideal; $f_{R}$ is the restriction of $\mathrm{f}$ on the ring $\mathrm{R}$.

(e) The AH-factor $R(I) /$ kerf is AHS - isomorphic to $f(R(I))$.

\section{Definition 2.13: [1]}

Let $\mathrm{R}(\mathrm{I})$ be a neutrosophic commutative ring and $P=P_{0}+P_{1} I$ be an AH-ideal, we say that:

(a) $\mathrm{P}$ is a weak prime $\mathrm{AH}$-ideal if $P_{0}, P_{1}$ are prime ideals in $\mathrm{R}$.

(b) $\mathrm{P}$ is a weak maximal $\mathrm{AH}$-ideal if $P_{0}, P_{1}$ are maximal ideals in $\mathrm{R}$.

(c) $\mathrm{P}$ is a weak principal $\mathrm{AH}$-ideal if $P_{0}, P_{1}$ are principal ideals in $\mathrm{R}$.

\section{Main concepts and discussion}

\section{Definition 3.1:}

Let $\left(\mathrm{R}\left(I_{1}, I_{2}\right),+, \times\right)$ be a refined neutrosophic ring, and $P_{0}, P_{1}, P_{2}$ be three ideals in the ring $\mathrm{R}$ then the set $P=\left(P_{0}, P_{1} I_{1}, P_{2} I_{2}\right)=\left\{\left(a, b I_{1}, c I_{2}\right) ; a \in P_{0}, b \in P_{1}, c \in P_{2}\right\}$ is called a refined neutrosophic AH-ideal.

If $P_{0}=P_{1}=P_{2}$ then $\mathrm{P}$ is called a refined neutrosophic AHS-ideal.

\section{Definition 3.2:}

Let $(\mathrm{R},+, \times),(\mathrm{T},+, \times)$ be two rings and $f_{R}: R \rightarrow T$ is a homomorphism : 
The map $f: \mathrm{R}\left(I_{1}, I_{2}\right) \rightarrow \mathrm{T}\left(I_{1}, I_{2}\right) ; f\left(x, y I_{1}, z I_{2}\right)=\left(f_{R}(x), f_{R}(y) I_{1}, f_{R}(z) I_{2}\right)$ is called a refined AHS-homomorphism.

It is easy to see that for all $x, y \in \mathrm{R}\left(I_{1}, I_{2}\right)$, we have $f(x+y)=f(x)+f(y), f(x \times y)=f(x) \times f(y)$.

\section{Example 3.3:}

Suppose that $\mathrm{R}=\left(Z_{6},+, \times\right), T=\left(Z_{10},+, \times\right)$ are two rings and $f_{R}: R \rightarrow T ; f(a)=5 a$ is homomorphism, the related refined AHS-homomorphism can be defined:

$f: \mathrm{R}\left(I_{1}, I_{2}\right) \rightarrow \mathrm{T}\left(I_{1}, I_{2}\right) ; f\left(x, y I_{1}, z I_{2}\right)=\left(5 x, 5 y I_{1}, 5 z I_{2}\right)$.

The previous example shows that refined AH-homomorphism is not a refined neutrosophic homomorphism in general because:

$f\left(I_{1}\right) \neq I_{1}$

Definition 3.4:

(a) Let $f: \mathrm{R}\left(I_{1}, I_{2}\right) \rightarrow \mathrm{T}\left(I_{1}, I_{2}\right)$ be a refined AHS-homomorphism, we define refined AH-Kernel of $f$ by:

$\mathrm{AH}-\operatorname{Kerf}=\left\{\left(a, b I_{1}, c I_{2}\right) ; a, b, c \in \operatorname{Kerf}_{R}\right\}=\left(\operatorname{Kerf}_{R}, \operatorname{Kerf}_{R} I_{1}, \operatorname{Kerf}_{R} I_{2}\right)$.

(b) Let $\mathrm{S}=\left(S_{0}, S_{1} I_{1}, S_{2} I_{2}\right)$ be a subset of $\mathrm{R}\left(I_{1}, I_{2}\right)$, then: $f(S)=\left(f_{R}\left(S_{0}\right), f_{R}\left(S_{1}\right) I_{1}, f_{R}\left(S_{2}\right) I_{2}\right)=$ $\left\{\left(f_{R}\left(a_{0}\right), f_{R}\left(a_{1}\right) I_{1}, f_{R}\left(a_{2}\right) I_{2}\right) ; a_{i} \in S_{i}\right\}$.

(c) Let $\mathrm{S}=\left(S_{0}, S_{1} I_{1}, S_{2} I_{2}\right)$ be a subset of $\mathrm{T}\left(I_{1}, I_{2}\right)$, then:

$$
f^{-1}(S)=\left(f_{T}^{-1}\left(S_{0}\right), f_{T}^{-1}\left(S_{1}\right) I_{1}, f_{T}^{-1}\left(S_{2}\right) I_{2}\right) .
$$

\section{Definition 3.5:}

Let $f: \mathrm{R}\left(I_{1}, I_{2}\right) \rightarrow \mathrm{T}\left(I_{1}, I_{2}\right)$ be a refined AHS-homomorphism we say that $f$ is a refined AHS-isomorphism if it is a bijective map, $\mathrm{R}\left(I_{1}, I_{2}\right), \mathrm{T}\left(I_{1}, I_{2}\right)$ are called AHS-isomorphic refined neutrosophic rings.

It is easy to see that restriction $f_{R}$ will be an isomorphism between $\mathrm{R}, \mathrm{T}$.

\section{Theorem 3.6:}

Let $f: \mathrm{R}\left(I_{1}, I_{2}\right) \rightarrow \mathrm{T}\left(I_{1}, I_{2}\right)$ be a refined AHS-homomorphism we have:

(a) $A H-\operatorname{Ker} f$ is a refinedneutrosophic AHS-ideal of $\mathrm{R}\left(I_{1}, I_{2}\right)$.

(b) If $\mathrm{P}$ is a refined neutrosophic AH-ideal of $\mathrm{R}\left(I_{1}, I_{2}\right), f(P)$ is a refined neutrosophic AH-ideal of $\mathrm{T}\left(I_{1}, I_{2}\right)$.

(c) If $\mathrm{P}$ is a refined neutrosophic AHS-ideal of $\mathrm{R}\left(I_{1}, I_{2}\right), f(P)$ is a refined neutrosophic AHS-ideal of $\mathrm{T}\left(I_{1}, I_{2}\right)$.

Proof:

(a) Since $\operatorname{Ker} f_{R}$ is an ideal of R,AH - $\operatorname{kerf}=\left(\operatorname{Ker} f_{R}, \operatorname{Kerf}_{R} I_{1}, \operatorname{Kerf}_{R} I_{2}\right)$ is a refined neutrosophic AHS-ideal of $\mathrm{R}\left(I_{1}, I_{2}\right)$.

(b)Suppose that $P=\left(P_{0}, P_{1} I_{1}, P_{2} I_{2}\right)$ is a refined neutrosophic AH-ideal of $\mathrm{R}\left(I_{1}, I_{2}\right)$. Since $f_{R}\left(P_{i}\right)$ is an ideal of $\mathrm{T}, f(P)=\left(f_{R}\left(P_{0}\right), f_{R}\left(P_{1}\right) I_{1}, f_{R}\left(P_{2}\right) I_{2}\right)$ is a refined neutrosophic AH-ideal. 
(c) The proof is similar to (b).

\section{Definition 3.7:}

Let $\left(\mathrm{R}\left(I_{1}, I_{2}\right),+, \times\right)$ be a refined neutrosophic ring and $\mathrm{P}=\left(P_{0}, P_{1} I_{1}, P_{2} I_{2}\right)$ be a refined neutrosophic AH-ideal then:

(a) We say that $\mathrm{P}$ is a weak prime refined neutrosophic $\mathrm{AH}$-ideal if $P_{i} ; i \in\{0,1,2\}$ are prime ideals in $\mathrm{R}$.

(b) We say that $\mathrm{P}$ is a weak maximal refined neutrosophic $\mathrm{AH}$-ideal if $P_{i} ; i \in\{0,1,2\}$ are maximal ideals in $\mathrm{R}$.

(c) We say that $\mathrm{P}$ is a weak principal refined neutrosophic $\mathrm{AH}$-ideal if $P_{i} ; i \in\{0,1,2\}$ are principal ideals in $\mathrm{R}$.

(d) We define the refined neutrosophic AH-factor as:

$$
\mathrm{R}\left(\mathrm{I}_{1}, \mathrm{I}_{2}\right) / \mathrm{P}=\left(\mathrm{R} / \mathrm{P}_{0}, \mathrm{R} / \mathrm{P}_{1} \mathrm{I}_{1}, \mathrm{R} / \mathrm{P}_{2} \mathrm{I}_{2}\right)=\left\{\left(\left[x_{0}+\mathrm{P}_{0}\right],\left[\mathrm{x}_{1}+\mathrm{P}_{1}\right] \mathrm{I}_{1},\left[\mathrm{x}_{2}+\mathrm{P}_{2}\right] \mathrm{I}_{2}\right) ; x_{0}, x_{1}, x_{2} \in R\right\}
$$

\section{Theorem 3.8:}

Let $f: \mathrm{R}\left(I_{1}, I_{2}\right) \rightarrow \mathrm{T}\left(I_{1}, I_{2}\right)$ be a refined AHS-homomorphism and $\mathrm{P}=\left(P_{0}, P_{1} I_{1}, P_{2} I_{2}\right)$ be a refined neutroosphic AH-ideal of $\mathrm{R}\left(\mathrm{I}_{1}, \mathrm{I}_{2}\right)$, let $\mathrm{Q}=\left(Q_{0}, Q_{1} I_{1}, Q_{2} I_{2}\right) \neq \mathrm{T}\left(I_{1}, I_{2}\right)$ be a refined neutrosophic AH-ideal of $\mathrm{T}\left(I_{1}, I_{2}\right)$, assume that $\operatorname{Kerf} f_{R} \leq P_{i} \neq R$ then:

(a) $\mathrm{P}$ is a weak prime refined neutrosophic AH-ideal of $\mathrm{R}\left(\mathrm{I}_{1}, \mathrm{I}_{2}\right)$ if and only if $f(P)$ is a weak primerefinedneutrosophic AH-ideal in $\mathrm{f}\left(\mathrm{R}_{(}\left(\mathrm{I}_{1}, \mathrm{I}_{2}\right)\right)$.

(b) $\mathrm{P}$ is a weak maximal AH-ideal of $\mathrm{R}\left(\mathrm{I}_{1}, \mathrm{I}_{2}\right)$ if and only if $f(P)$ is a weak maximal in $\mathrm{f}\left(\mathrm{R}\left(\mathrm{I}_{1}, \mathrm{I}_{2}\right)\right.$ ).

(c) Q is a weak prime AH-ideal of $\mathrm{T}\left(\mathrm{I}_{1}, \mathrm{I}_{2}\right)$ if and only if $f^{-1}(\mathrm{Q})$ is a weak prime in $\mathrm{R}\left(\mathrm{I}_{1}, \mathrm{I}_{2}\right)$.

(d) Q is a weak maximal AH-ideal of $\mathrm{T}\left(\mathrm{I}_{1}, \mathrm{I}_{2}\right)$ if and only if $f^{-1}(\mathrm{Q})$ is a weak maximal in $\mathrm{R}\left(\mathrm{I}_{1}, \mathrm{I}_{2}\right)$.

Proof:

The proof is similar to the Theorem 3.15 in [1].

It is easy to see that conditions (a), (b) are still true if P is an AHS-ideal and conditions (c), (d) are still true if Q is an AHS-ideal.

\section{Theorem 3.9:}

The refined neutrosophic $\mathrm{AH}$-factor $\mathrm{R}\left(\mathrm{I}_{1}, \mathrm{I}_{2}\right) / \mathrm{P}$ is a ring with respect to the following operations:

Let $x=\left(x_{0}+P_{0},\left(x_{1}+P_{1}\right) I_{1},\left(x_{2}+P_{2}\right) I_{2}\right), y=\left(y_{0}+P_{0},\left(y_{1}+P_{1}\right) I_{1},\left(y_{2}+P_{2}\right) I_{2}\right)$, be two arbitrary elements in $\mathrm{R}\left(I_{1}, I_{2}\right)$ then:

$x+y=\left(\left[x_{0}+y_{0}\right]+P_{0},\left(\left[x_{1}+y_{1}\right]+P_{1}\right) I_{1},\left(\left[x_{2}+y_{2}\right]+P_{2}\right) I_{2}\right)$,

$x \times y=\left(\left[x_{0} \times y_{0}\right]+P_{0},\left(\left[x_{1} \times y_{1}\right]+P_{1}\right) I_{1},\left(\left[x_{2} \times y_{2}\right]+P_{2}\right) I_{2}\right)$.

Proof:

The proofis similar to the Theorem 3.9 in [1].

\section{Example 3.10:}


Let $\mathrm{R}=\left(Z_{6},+, \times\right), \mathrm{T}=\left(Z_{10},+, \times\right)$ be two rings, and $f$ be the refined neutrosophic AHS-homomorphism defined in Example 3.3, we have the following:

(a) $P_{0}=\{0,2,4\}, P_{1}=\{0,3\}$ are two ideals in $Z_{6}$ thus $\mathrm{P}=\left(P_{0}, P_{0} I_{1}, P_{1} I_{2}\right)$ is a refined neutrosophic AH-ideal of $\mathrm{R}\left(\mathrm{I}_{1}, \mathrm{I}_{2}\right)$.

(b) $f(P)=\left(f\left(P_{0}\right), f\left(P_{0}\right) I_{1}, f\left(P_{1}\right) I_{2}\right)=\left\{(0,0,0),\left(0,0,5 I_{2}\right)\right\}$ is a refined neutrosophic AH-ideal in $\mathrm{T}\left(\mathrm{I}_{1}, \mathrm{I}_{2}\right)$.

(c) $Q_{0}=\{0,2,4,6,8\}$ is a maximal ideal in $Z_{10}$ and $f_{T}^{-1}\left(Q_{0}\right)=\{0,2,4\}$, so $Q=\left(Q_{0}, Q_{0} I_{1}, Q_{0} I_{2}\right)$ is a weak maximal refined neutrosophic AHS-ideal in $\mathrm{T}\left(\mathrm{I}_{1}, \mathrm{I}_{2}\right)$, we have $f^{-1}(Q)=\left(\{0,2,4\},\{0,2,4\} I_{1},\{0,2,4\} I_{2}\right)$ is a weak maximal refined neutrosophic AHS-ideal in $\mathrm{R}\left(\mathrm{I}_{1}, \mathrm{I}_{2}\right)$.

\section{Example 3.11:}

(a) In the ring $(\mathrm{Z},+, \times), \mathrm{P}=<3>, \mathrm{Q}=<2>$ are two prime and maximal ideals, thus $\mathrm{M}=\left(\mathrm{P}, Q I_{1}, Q I_{2}\right)=\left\{\left(3 \mathrm{a}, 2 \mathrm{~b} I_{1}, 2 \mathrm{c} I_{2}\right)\right.$; $\mathrm{a}, \mathrm{b}, \mathrm{c} \in Z\}$ is a weak maximal/prime refined neutrosophic AH-ideal of $\left(\mathrm{Z}\left(I_{1}, I_{2}\right),+, \times\right)$.

(b)The map $f_{Z}: Z \rightarrow Z_{6} ; f(a)=a \bmod 6$ is a homomorphism so the related refined neutrosophic AHShomomorphism is

$f: \mathrm{Z}\left(\mathrm{I}_{1}, \mathrm{I}_{2}\right) \rightarrow \mathrm{Z}_{6}\left(\mathrm{I}_{1}, \mathrm{I}_{2}\right) ; f\left(a, b I_{1}, c I_{2}\right)=\left(\operatorname{amod} 6,(b \bmod 6) I_{1},(c \bmod 6) I_{2}\right), A H-k e r f=\left(6 \mathrm{Z}, 6 \mathrm{ZI} I_{1}, 6 \mathrm{ZI}_{2}\right) \leq M$ since $6 \mathrm{Z} \leq P, Q$.

(c) $f(M)=\left(\{0,3\},\{0,2,4\} I_{1},\{0,2,4\} I_{2}\right)$ is a weak maximal/prime refined neutrosophic AH-ideal of $\mathrm{Z}_{6}\left(\mathrm{I}_{1}, \mathrm{I}_{2}\right)$.

\section{Definition 3.12:}

A refined neutrosophic ring $\mathrm{R}\left(\mathrm{I}_{1}, \mathrm{I}_{2}\right)$ is called weak principal refined neutrosophic AH-ring if every refined neutrosophic AH-ideal is weak principal.

\section{Theorem 3.13:}

Let $\mathrm{R}$ be a principal ideal ring then $\mathrm{R}\left(\mathrm{I}_{1}, \mathrm{I}_{2}\right)$ is weak principal refined neutrosophic AH-ring.

Proof:

Let $\mathrm{P}=\left(P_{0}, P_{1} I_{1}, P_{2} I_{2}\right)$ be a refined neutrosophic AH-ideal of $\mathrm{R}\left(\mathrm{I}_{1}, \mathrm{I}_{2}\right)$. Since $P_{i}$ are ideals in $\mathrm{R}$ and then principal this implies that $\mathrm{P}$ is a weak refined neutrosophic $\mathrm{AH}$-ideal; thus $\mathrm{R}\left(\mathrm{I}_{1}, \mathrm{I}_{2}\right)$ must be weak principal refined neutrosophic AH-ring.

\section{Example 3.14:}

The ring $(\mathrm{Z},+, \times)$ is principal ideals ring; thus $\mathrm{Z}\left(\mathrm{I}_{1}, \mathrm{I}_{2}\right)$ is weak principal refined neutrosophic AH-ring.

\section{Definition 3.15:}

Let $\left(\mathrm{R}\left(I_{1}, I_{2}\right),+, \times\right)$ be a refined neutrosophic ring and $\mathrm{P}=\left(P_{0}, P_{1} I_{1}, P_{2} I_{2}\right), \mathrm{Q}=\left(Q_{0}, Q_{1} I_{1}, Q_{2} I_{2}\right)$ be two refined neutrosophic AH-ideals of $\mathrm{R}\left(I_{1}, I_{2}\right)$, then we define:

(a) $P \cap Q=\left(P_{0} \cap Q_{0},\left[P_{1} \cap Q_{1}\right] I_{1},\left[P_{2} \cap Q_{2}\right] I_{2}\right)$.

$$
\text { (b) } P+Q=\left(P_{0}+Q_{0},\left[P_{1}+Q_{1}\right] I_{1},\left[P_{2}+Q_{2}\right] I_{2}\right) .
$$


(c) $P \times Q=\left(P_{0} \times Q_{0},\left[P_{1} \times Q_{1}\right] I_{1},\left[P_{2} \times Q_{2}\right] I_{2}\right)$.

\section{Theorem 3.16:}

Let $\left(\mathrm{R}\left(I_{1}, I_{2}\right),+, \times\right)$ be a refined neutrosophic ring and $\mathrm{P}=\left(P_{0}, P_{1} I_{1}, P_{2} I_{2}\right), \mathrm{Q}=\left(Q_{0}, Q_{1} I_{1}, Q_{2} I_{2}\right)$ be two refined neutrosophic AH-ideals of $\mathrm{R}\left(I_{1}, I_{2}\right)$, then:

$P \cap Q, P+Q, P \times Q$ are refined neutrosophic AH-ideals of $\mathrm{R}\left(I_{1}, I_{2}\right)$.

Proof:

As a result of Theorem $2.5 \mathrm{in}[1]$, we have $P_{i}+Q_{i}, P_{i} \cap Q_{i}, P_{i} \times Q_{i}$ are ideals of R, thus the proof is complete.

\section{Remark 3.17:}

Theorem 3.16 is still true if $\mathrm{P}$ and $\mathrm{Q}$ are refined neutrosophic AHS-ideals.

\section{Example 3.18:}

Let $R\left(I_{1}, I_{2}\right)=Z_{8}\left(I_{1}, I_{2}\right)$ and $\mathrm{Q}=\{0,4\}, \mathrm{S}=\{0,2,4,6\}$ be two principal ideals in $\mathrm{R}$, then:

(a) $\mathrm{P}=\left(\mathrm{S}, \mathrm{Q} I_{1}, \mathrm{SI}_{2}\right)$ is a refined neutrosophic $\mathrm{AH}$-ideal of $R\left(I_{1}, I_{2}\right)$, the related refined neutrosophic $\mathrm{AH}$-factor is:

$R\left(I_{1}, I_{2}\right) / P=\left(R / S, R / Q I_{1}, R / S I_{2}\right)=\left(\{\mathrm{S}, 1+\mathrm{S}\},\{\mathrm{Q}, 1+\mathrm{Q}, 2+\mathrm{Q}, 3+\mathrm{Q}\} I_{1},\{\mathrm{~S}, 1+\mathrm{S}\} I_{2}\right)$.

To clarify addition and multiplication on $R\left(I_{1}, I_{2}\right) / P$ we take:

$x=\left(1+S,(1+Q) I_{1}, S I_{2}\right), y=\left(S,(2+Q) I_{1},(1+S) I_{2}\right)$, we have:

$x+y=\left([1+0]+S,([1+2]+Q) I_{1},([0+1]+S) I_{2}\right)=\left(1+\mathrm{S},(3+\mathrm{Q}) I_{1},(1+S) I_{2}\right)$.

$x \times y=\left([1 \times 0]+S,([1 \times 2]+Q) I_{1},([0 \times 1]+S) I_{2}\right)=\left(\mathrm{S},(2+\mathrm{Q}) I_{1}, S I_{2}\right)$.

\section{Conclusion}

In this article we defined concepts of refined neutrosophic AH-ideal/ AHS-ideal in a refined neutrosophic ring.We studied some of elementary properties of these concepts.Also, notions as weak maximal, prime and principal refined neutrosophic AH-ideal and refined AHS-homomorphisms were introduced and checked.

Funding: "This research received no external funding."

Conflicts of Interest: "The authors declare no conflict of interest."

\section{References}

[1].Abobala, M., "On Some Special Substructures of Neutrosophic Rings and Their Properties", International Journal of Neutrosophic Science", Vol4, pp 72-81, 2020.

[2].Adeleke, E.O., Agboola, A.A.A., and Smarandache, F., "Refined Neutrosophic Rings I", International Journal of Neutrosophic Science, Vol 2, pp 77-81, 2020.

[3].Adeleke, E.O., Agboola, A.A.A., and Smarandache, F., "Refined Neutrosophic Rings II", International Journal of Neutrosophic Science, Vol 2, pp 89-94, 2020. 
[4].Agboola, A.A.A., Adeleke, E.O., and Akinlye, S.A., "Neutrosophic Rings II ",International J.Mathcombin, $\operatorname{vol}(2)$, pp 1-8, 2012.

[5].Agboola, A.A.A., Akinola, A.D., and Oyebola, O.Y.," NeutrosophicRings I ", International J.Mathcombin, $\operatorname{vol}(4)$, pp 1-14, 2011.

[6].Agboola, A.A.A., and Davvaz, B.," On Neutrosophic Canonical Hypergroups and NeutrosophicHyperrings", Neutrosphic Sets and Systems, Vol 2, pp 34-41, 2014.

[7].Chalapathi, T., Kumar, K., "Neutrosophic Graphs of Finite Groups", Neutrosophic Sets and Systems, vol 15, pp22-30, 2017.

[8].Chalabathi, T., and Kumar, K.,"Neutrosophic Units of Neutrosophic Rings and Fields", Neutrosophic Sets and Systems, vol 21, pp 5-9, 2018.

[9].Haushi, M., "Algebraic Structures 2 ", Tishreen University Press, pp 132-190, 2004.

[10].Kandasamy, V.W.B., and Smarandache, F., "Some Neutrosophic Algebraic Structures and Neutrosophic NAlgebraic Structures", Hexis, Phonex, Arizona 2006. 